\title{
Exciting Gauge Field and Gravitons in Brane-Antibrane Annihilation
}

\author{
Anupam Mazumdar ${ }^{1,2}$ and Horace Stoica ${ }^{3}$ \\ ${ }^{1}$ Physics Department, Lancaster University, LA1 4YB, United Kingdom \\ ${ }^{2}$ Niels Bohr Institute, Copenhagen University, Blegdamsvej-17, DK-2100, Denmark \\ ${ }^{3}$ Theoretical Physics, Blackett Laboratory, Imperial College, London, SW7 2AZ, United Kingdom
}

(Received 1 August 2008; published 5 March 2009)

\begin{abstract}
In this Letter we point out the inevitability of an explosive production of gauge field and gravity wave during an open string tachyon condensation in a cosmological setting, in an effective field theory model. We will be particularly studying a toy model of brane-antibrane inflation in a warped throat where inflation ends via tachyon condensation. We point out that a tachyonic instability helps fragmenting the homogeneous tachyon and excites gauge field and contributes to the stress-energy tensor which also feeds into the gravity waves.
\end{abstract}

PACS numbers: 11.25.Wx, 04.30.Tv, 04.60.Cf, 98.80.Cq

There are many cosmological sources of gravity wave generation; for a review see [1]. In this Letter we will show how an interesting scenario emerges in string cosmology where there exists a plethora of examples of open string tachyon condensation [2]. We will show how gravity waves and gauge field are excited in a field theory model of braneantibrane annihilation particularly in a cosmological setting.

The tachyon plays an important role in terminating brane-antibrane inflation [3], for a review see [4], when a pair comes close to each other, less than a critical distance. When the branes are far apart the tachyon field does not play any role, while the brane-antibrane separation plays the role of an inflaton and the brane tension generate the vacuum energy density. The slow attraction of the branes towards each other gives rise to a primordial inflation and also a graceful exit. In this process the so called tachyon field acts almost like a waterfall field like in the case of a hybrid inflation [5], where the (mass) ${ }^{2}$ of the waterfall field changes from being positive to zero to negative. In all these examples the waterfall field and the tachyon in string theory examples are considered to be a homogeneous field evolving with time.

In this Letter we discuss a couple of important consequences of a brane-antibrane inflation (although the end of inflation via brane-antibrane lends the greatest support for studying the tachyons, but open string tachyons could also give rise to primordial inflation [6], and furthermore tachyons can possibly generate large non-Gaussianity [7]) in the field theory model. Note that field theoretical treatment is an oversimplification of the brane annihilation process, but we will be content with it as a toy model to investigate excitation of gravity wave and gauge field. It would be interesting to see how stringy effects will modify our findings.

First, in a cosmological context the tachyon will never keep the homogeneity, the tachyon will be fragmented by virtue of inhomogeneity created by the quantum fluctuations, quite similar to the tachyonic preheating [8]. Second, the tachyon in string theory couples bilinearly to the gauge fields residing on the world volume of the branes. It can be shown by field redefinitions that in an Einstein frame the gauge fields couple minimally, therefore, the gauge fields are made into Higgs fields by the tachyon, i.e., the gauge fields obtain vacuum expectation value (VEV) dependent masses. This is an ideal case when a time dependent VEV can excite gauge fields at sub- and super-Hubble scales.

Third, the fragmentation of the tachyon and its inhomogeneous perturbations seeds the anisotropic stress tensor which leads to gravity wave generation. The frequency of these gravity waves depends on the string scale which we take to be close to $M_{s} \sim 10^{14} \mathrm{GeV}$ (below the scale of grand unified theory). Since the scale is quite high, therefore the frequency will not be detectable by future gravity wave experiments.

In particularly, we will be studying inflation in a warped throat, while the SM or the MSSM fields are located in a different throat with a different warping. For simplicity, we will consider a simple $D 3-\overline{D 3}$ system, where the modulus determining the interbrane separation drives inflation and inflation ends via tachyon condensation. Inflation in such a setup will not directly reheat the MSSM throat [9], reheating happens indirectly via moduli belonging to the MSSM [10]; see also [11]. One plausible solution is to inflate the universe within the MSSM throat [12], which would naturally reheat the universe with the MSSM degrees of freedom. However, note that the branes do carry gauge fields in their world volume; the tachyon in a brane-antibrane system is a bifundamental field that couples only to a linear combination of the two gauge fields. These gauge fields drop out of the dynamics once the pair has annihilated. However, more realistic models would include a stack of $N$ (anti)branes located at the bottom of the throat and a number of $M$ mobile branes moving towards them. After annihilation the remaining stack of branes supports an unbroken $U(N-M)$ [13-15]. The original $U(N) \times$ $U(M)$ is made into Higgs fields down to $U(N-M)$ when the tachyon develops a VEV at the end of inflation. 
When the brane and the antibrane are coincident the action for the tachyon field responsible for the anniliation of the pair is described by $[16,17]$

$$
\begin{aligned}
\mathcal{S}= & -2 T_{D 3} \int \sqrt{-g_{4}} d^{4} x e^{-2 \pi \alpha^{\prime} \mathrm{T} \overline{\mathrm{T}}}\left[1+8 \pi\left(\alpha^{\prime}\right)^{2}\right. \\
& \left.\times \ln (2) D^{\mu} \bar{T} D_{\mu} T+\frac{\gamma \alpha^{\prime 2}}{8}\left(F_{\mu \nu}^{+}-F_{\mu \nu}^{-}\right)^{2}\right] .
\end{aligned}
$$

Here $F^{+}$and $F^{-}$are the gauge fields that live in the world volume of the brane and antibrane, respectively. The tachyon is a bifundamental field that couples only to a linear combination of the two gauge fields $D_{\mu} T=\partial_{\mu} T-$ $\left(A_{\mu}^{+}-A_{\mu}^{-}\right) T$. If we expand the exponential term as $e^{-2 \pi \alpha^{\prime} \mathrm{TT}}=1-2 \pi \alpha^{\prime} \mathrm{T} \overline{\mathrm{T}}$ and write the $D 3$-brane tension as $T_{D 3}=1 /\left[(2 \pi)^{3}\left(\alpha^{\prime}\right)^{2} g_{s}\right]$. The numerical prefactor $8 \pi \ln (2)$ can be absorbed in a field redefinition and the action with $\chi=(\sqrt{2 \ln 2} r / \pi R) T$ can be brought to the form (the scalar part only). To find the warped tachyon mass we write the metric in the warped throat takes the form (up to corrections in a KS type throat) $d s_{10}^{2}=$ $\frac{r^{2}}{R^{2}} d s_{4}^{2}+\frac{R^{2}}{r^{2}}\left(d r^{2}+r^{2} d \Omega_{5}^{2}\right)$; see Ref. [18]:

$$
\begin{aligned}
S & =\int d^{4} x\left(\frac{2 r^{4}}{R^{4}} T_{D 3}+\frac{1}{2} D_{\mu} \chi D^{\mu} \chi-\frac{r^{2}}{R^{2}} \frac{M_{s}^{2}}{2 \ln (2)} \chi^{2}\right) \\
& =\int d^{4} x\left(\Lambda_{4}+\frac{1}{2} D_{\mu} \chi D^{\mu} \chi-\frac{1}{2} m_{T}^{2} \chi^{2}\right) .
\end{aligned}
$$

Denoting $r / R=w$, the warp factor, we see that the cosmological constant given by the brane tension and the tachyon mass depend only on the warped string scale $m=$ $w M_{s}$, and therefore the Hubble constant during inflation and the tachyon mass during reheating are related $\Lambda_{4}=$ $2 w^{4} T_{D 3}=w^{4} 2 M_{s}^{4} /(2 \pi)^{3} g_{s}=2 m^{4} /(2 \pi)^{3} g_{s}$.

In order to understand the aftermath of inflation, we need to include the gauge fields and we also need to model the scalar potential in the tachyonic phase by a polynomial function of the fields.

$$
\begin{aligned}
\mathcal{L}= & \frac{1}{2} \partial_{\mu} \phi \partial^{\mu} \phi+\frac{1}{2} D_{\mu} \chi D^{\mu} \chi^{*}+\frac{1}{2} m_{\text {inflaton }}^{2} \phi^{2} \\
& +\frac{1}{2} m_{T}^{2}|\chi|^{2}\left(\phi^{2}-\phi_{0}^{2}\right)+\frac{\lambda}{4}|\chi|^{4}-\frac{1}{4} F^{2} .
\end{aligned}
$$

The value of $\phi_{0}$ represents the critical distance at which the tachyon field becomes unstable. We had to add a quartic term for the tachyon potential, so that the overall potential is bounded from below. Otherwise, the simulation cannot be performed. We are interested in the dynamics of the tachyon at the top of its potential, $\chi \approx 0$, where the quartic term is negligible.

We have verified that the quartic term does not change the results of our simulations as far as the tachyonic phase is concerned. Making the quartic term smaller will result in a potential with deeper minima, and therefore a longer tachyonic phase. However, since the VEV of the field increases exponentially with time, the duration of the tachyonic phase depends only logarithmically in the coefficient of the quartic term. The quartic term has a large influence during the turbulent phase after the tachyonic amplification has ended. Since the minimum is deeper, the field will reach this minimum with higher kinetic energy, and therefore more energy is transferred to the gravity waves in a given time interval.

The Lagrangian in Eq. (1) describes the brane-antibrane system only when the brane and the antibrane are coincident. In our approximate Lagrangian, Eq. (3), we include the dependence of the tachyon mass on the inflaton VEV, that is the brane-antibrane separation.

To study the gravity waves, we follow the approach of [19] were the perturbations of the Einstein equations are studied. We start by considering the metric fluctuations: $d s^{2}=-d t^{2}+a^{2}(t)\left(\delta_{i j}+h_{i j}\right) d x^{i} d x^{j}$. The corresponding equation for the fluctuations of the metric reads: $\square h_{i j}=$ $16 \pi G \delta T_{i j}$, where $T_{i j}$ is the anisotropic stress-energy tensor. Just as in [19] we follow the evolution of only the traceless part of the tensor fluctuations: $\ddot{h}_{i j}-\nabla^{2} h_{i j}=$ $16 \pi G \Pi_{i j}$, where $\Pi_{i j}=T_{i j}-\frac{1}{3} T \delta_{i j}$. We may neglect the expansion of the universe since $M_{s} \gg H$ at the time when tachyon is rolling after inflation.

The stress-energy tensor gets contributions from both the charged tachyon and uncharged inflaton, as well as the gauge fields. Details of the calculation of the stress-energy tensor will be presented in a separate paper. Here we simply quote the results:

$$
\begin{aligned}
\Pi_{i j}= & F_{i C} F_{j}{ }^{C}-\frac{1}{3} \delta_{i j} F_{k C} F^{k C}-D_{i} \chi D_{j} \chi^{*}+\frac{1}{3} \delta_{i j} D_{k} \chi D^{k} \chi^{*} \\
& -\partial_{i} \phi \partial_{j} \phi^{*}+\frac{1}{3} \delta_{i j} \partial_{k} \phi \partial^{k} \phi^{*} .
\end{aligned}
$$

When studying the effects of the gauge fields we look at both the energy the gauge fields pick up during the evolution as well as their contribution as a source for the gravitational waves. The energy density of the gravitational waves is simply the $t_{00}$ component of the energymomentum tensor of the gravitational waves: $t_{00}=\frac{1}{32 \pi G} \times$ $\left\langle\partial_{0} h_{i j}^{\mathrm{TT}} \partial_{0} h_{\mathrm{TT}}^{i j}\right\rangle$ where TT stands for the transverse-traceless part of the tensor fluctuations. The energy density calculated simply for $h_{i j}$ is related to that for $h_{i j}^{\mathrm{TT}}$ by a numerical factor given by the angular integral of the projection operator that transforms $h_{i j}$ into $h_{i j}^{\mathrm{TT}}$. We also use the synchronous gauge for the metric, so the $h_{00}$ and the $h_{0 i}$ components of the metric fluctuations are set to zero.

The parameters of the model are set to reflect the typical values for an inflationary model. We set the charge coupling the gauge field to the tachyon to 1 , and the same for the quartic self-coupling of the tachyon, $\lambda=1$. The mass of the inflaton is taken to be $m_{\phi}^{2}=0.01$, much smaller than the tachyon mass. We set the Newton constant to a value corresponding to the typical string mass we obtain by requiring that the brane-antibrane inflationary model reproduces the observed amplitude of the CMB fluctuations, $M_{S} / M_{P}=10^{-4}[4,15,20]$. This value for the ratio between 
the string and Planck scales tells us that all the modes in our simulation are inside the Hubble horizon. The Hubble constant during, and shortly after inflation is: $H^{2}=$ $V / 3 M_{P}^{2} \simeq M_{S}^{4} / 3 M_{P}^{2}=\left(M_{S} / M_{P}\right)^{2} M_{S}^{2} / 3$. We can now see how the Hubble radius compares with the size of our simulation box $\sim 30 l_{S}$, taking into account that the lattice spacing is about half the string length $l_{S}=1 / M_{S}: \frac{1}{H} \simeq$ $\frac{M_{P}}{M_{S}} l_{S}=10^{4} l_{S} \gg 30 l_{S}$. The value of the tachyon mass, controlled by the coupling of the tachyon to the inflaton field, is chosen to take 4 values and we compare the production of gravitational waves when the tachyon mass is changed. The time scale for the instability to grow depends on inversely proportional to the mass scale of the tachyon, therefore, a larger massive tachyon leads to a quicker exponential growth in gauge field and in gravitational waves compared to the smaller one; see Fig. 3.

For the initial condition, we assume that the tachyon mass is effectively vanishing towards the end of inflation where the inflaton VEV is $\approx \phi_{0}$. We then include initial fluctuation of the tachyon around this VEV with a spectrum given by the fluctuations of a scalar in the deSitter phase: $\chi(k)=c_{k} / k^{3 / 2}$. The Fourier coefficients of the field fluctuations had been taken in [19] to follow a Gaussian distribution with a dispersion dependent on the wave number as $\sigma_{k}^{2} \sim k^{-3}$. Here we will approximate the Gaussian distribution with a uniform one in the interval $\left(-1 / 2 k^{3 / 2}\right.$, $\left.1 / 2 k^{3 / 2}\right)$. We obtain this distribution by choosing $c_{k}$ uniformly distributed in the interval $(-1 / 2,1 / 2)$ and then using $c_{k} / k^{3 / 2}$ as the Fourier coefficients for the tachyon, thus the real and imaginary parts are being independent random numbers.

The inflaton starts with a VEV of $|\phi|=\phi_{0}$, corresponding the onset of the tachyonic instability. On top of this VEV we superimpose fluctuations corresponding to the deSitter phase, just like for the tachyon. The gauge fields have only the fluctuations corresponding to the deSitter phase. We take the gravitational waves to start at varying amplitude, see Fig. 1, all of them saturate to a similar amplitude. The time is measured in a string unit.

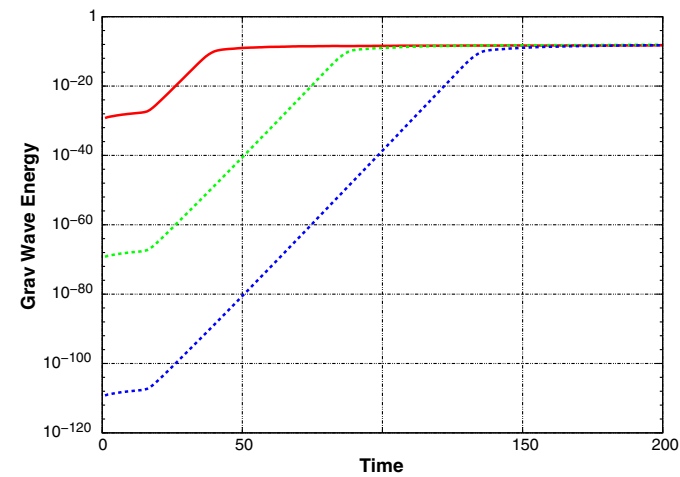

FIG. 1 (color online). Gravity wave energy with varying initial amplitudes. Note that different initial amplitudes saturate at a similar value for a fixed tachyon mass $\sim M_{s} / 2$.
In Fig. 2 we have shown various energy components of the tachyon. The potential energy of the tachyon is negative given our choice of a potential. We observe that the production of gravitational waves does not start until the gradient energy (dark blue curve) of the tachyon starts to pick up, see Figs. 2 and 3. The first "spike" in the energy graph, see Fig. 3, corresponds to the first oscillation of the tachyon, but the field stays almost homogeneous during this first oscillation. When the gradient energy picks up and the field condensate fragments, then only the gravity waves are produced and also the gauge field is excited. The energy in the $\phi$ field remains negligible.

The energy stored in the gauge field also gets exponentially amplified due to the fragmentation of the tachyon field. Although they carry most of the energy, see Fig. 3, compared to the gravity waves, their amplification happens at a slightly later stage; i.e., compare the two in Fig. 3 for tachyon mass $M_{s} / 2$.

In Figs. 4 we have shown 4 snapshots of the isosurface of the constant energy density for the gauge field, the gravity waves, the tachyon and the inflaton. Couple of points to note here, all the fields except the inflaton shows a remarkable departure in the homogeneity. Except the inflaton all fields undergo long wavelength excitations (they all look relatively smooth on small scales), while the inflaton obtains the largest inhomogeneity on the smaller scales. This is due to the fact that the there is no long wavelength amplification for the inflaton, rather the inflaton starts oscillating rapidly with an enhanced frequency by virtue of the coupling $\sim m_{T}^{2}|\chi|^{2}$. However, as the tachyon fragments, the $\chi$ field no longer retains the homogeneity, therefore the inflaton also obtains space dependent mass which fragments the inflaton on sub-Hubble scales.

Let us now comment on the gravity waves, its amplitude and frequency. Because of the tachyonic growth the amplitude of the frequency is given by $\rho_{\mathrm{GW}} / \rho_{0} \sim t_{00} / M_{S}^{4} \sim$ $\left\langle\dot{h}_{i j} \dot{h}^{i j}\right\rangle_{V}$, where $V$ stands for the volume average. From

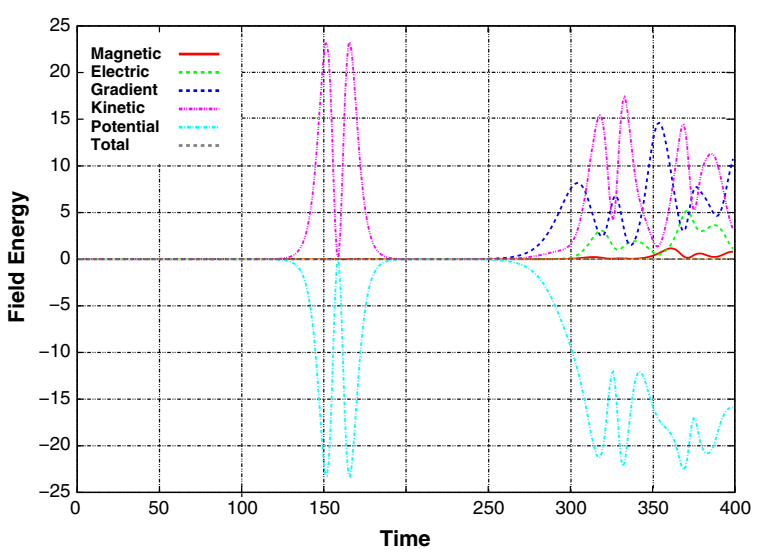

FIG. 2 (color online). The energy components of the tachyon and gauge field for the tachyon mass $M_{S} / 2$. Note that the total energy remains conserved. 


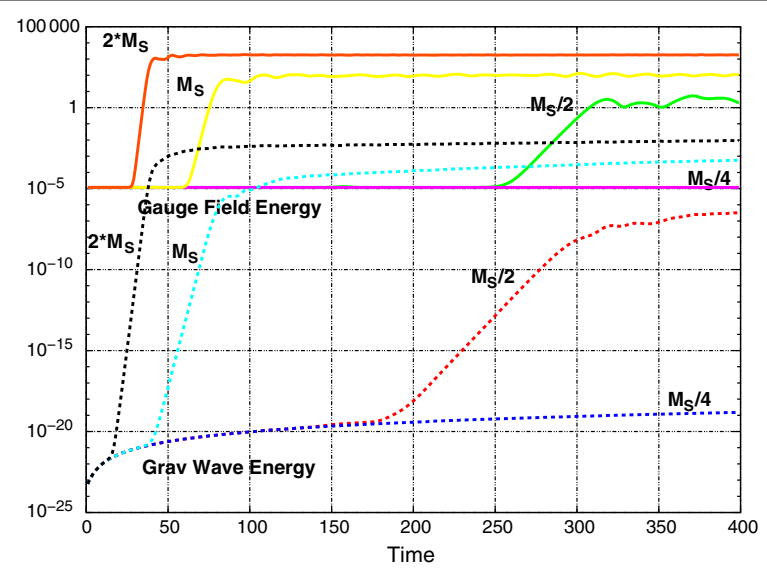

FIG. 3 (color online). The energy pumped into the gauge field and the gravity waves.

Fig. 3 it is clear that the factor $\rho_{\mathrm{GW}} / \rho_{0} \sim 10^{-6}$ while its frequency depends on the details of the expansion history of the universe, however, no matter what happens lately, the frequency will be well beyond the reach of the future gravity wave detectors.

To summarize, we have shown a brane-antibrane annihilation inevitably ends up in fragmentation of a tachyon which excites gauge field and gravity waves simultaneously. We also note that the excitations are generically long wavelength in nature, except that of the inflaton. However our lattice box size is such that we can only explore sub-Hubble processes. The amplification of gauge and gravity waves depends on the mass of a tachyon. A larger mass tachyon leads to a quicker growth as compared to a smaller tachyon mass. The amplitude of the gravity waves could be quite large, i.e., $\rho_{\mathrm{GW}} / \rho_{0} \sim 10^{-6}$ but their frequency will be towards the high end of the spectrum where future detection will not be foreseeable. Our simulation also unveils the first study of exciting gravity waves where the gauge field has been taken into account. We found that the initial burst of gravity waves arises from the fragmentation of the tachyon field, at only later stages the gauge field feeds into the production of gravity waves. One point to note here that we have not studied turbulence of the gauge field and the gravity waves, which will be considered in a separate publication.
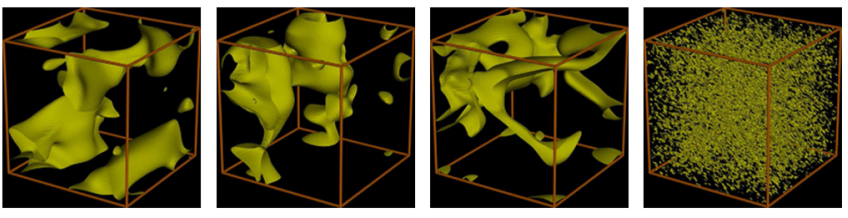

FIG. 4 (color online). Snapshots of isosurface of the energy density for the gauge field, gravity waves, tachyon and the inflaton (from left to right) at $t=300$.
The numerical simulations have been done on the Imperial College HPC cluster. A. M. is partly supported by grant (MRTN-CT-2006-035863).

[1] M. Maggiore, Phys. Rep. 331, 283 (2000).

[2] A. Sen, J. High Energy Phys. 04 (2002) 048.

[3] C. P. Burgess et al., J. High Energy Phys. 07 (2001) 047; C. P. Burgess, J.M. Cline, H. Stoica, and F. Quevedo, J. High Energy Phys. 09 (2004) 033; N. T. Jones, H. Stoica, and S.H.H. Tye, J. High Energy Phys. 07 (2002) 051; S. Kachru et al., J. Cosmol. Astropart. Phys. 10 (2003) 013; J. M. Cline and H. Stoica, Phys. Rev. D 72, 126004 (2005); D. Baumann et al., J. Cosmol. Astropart. Phys. 01 (2008) 024.

[4] L. McAllister and E. Silverstein, Gen. Relativ. Gravit. 40, 565 (2008); R. Kallosh, Lect. Notes Phys. 738, 119 (2008).

[5] A. D. Linde, Phys. Rev. D 49, 748 (1994).

[6] A. Mazumdar, S. Panda, and A. Perez-Lorenzana, Nucl. Phys. B614, 101 (2001).

[7] K. Enqvist et al., J. High Energy Phys. 08 (2005) 084.

[8] G. N. Felder et al., Phys. Rev. Lett. 87, 011601 (2001); G. N. Felder, L. Kofman, and A. D. Linde, Phys. Rev. D 64, 123517 (2001); G. N. Felder and L. Kofman, Phys. Rev. D 75, 043518 (2007).

[9] L. Kofman and P. Yi, Phys. Rev. D 72, 106001 (2005); D. Chialva, G. Shiu, and B. Underwood, J. High Energy Phys. 01 (2006) 014.

[10] A. R. Frey, A. Mazumdar, and R. C. Myers, Phys. Rev. D 73026003 (2006); A. Buchel and L. Kofman, arXiv:0804.0584.

[11] R.H. Brandenberger, A. R. Frey, and S. Kanno, Phys. Rev. D 76, 083524 (2007); R. H. Brandenberger, A. R. Frey, and L.C. Lorenz, arXiv:0712.2178. R.H. Brandenberger, K. Dasgupta, and A.C. Davis, arXiv:0801.3674.

[12] R. Allahverdi et al., Phys. Rev. Lett. 97, 191304 (2006); R. Allahverdi et al., J. Cosmol. Astropart. Phys. 06 (2007) 019; R. Allahverdi, A. Kusenko, and A. Mazumdar, J. Cosmol. Astropart. Phys. 07 (2007) 018.

[13] P. Horava, Adv. Theor. Math. Phys. 2, 1373 (1999).

[14] E. Witten, J. High Energy Phys. 12 (1998) 019.

[15] S. Sarangi and S. H. Tye, Phys. Lett. B 536, 185 (2002).

[16] P. Kraus and F. Larsen, Phys. Rev. D 63, 106004 (2001).

[17] T. Takayanagi, S. Terashima, and T. Uesugi, J. High Energy Phys. 03 (2001) 019.

[18] D. Cremades, F. Quevedo, and A. Sinha, J. High Energy Phys. 10 (2005) 106.

[19] J. Garcia-Bellido, D. G. Figueroa, and A. Sastre, arXiv:0707.0839.J. Garcia-Bellido and D. G. Figueroa, arXiv:0801.4109.J.F. Dufaux et al., Phys. Rev. D 76 123517 (2007).

[20] R. A. Battye, B. Garbrecht, A. Moss, and H. Stoica, J. Cosmol. Astropart. Phys. 01 (2008) 020; N. T. Jones, H. Stoica, and S. H. H. Tye, Phys. Lett. B 563, 6 (2003). 